\title{
Response order effects in the Youth Tobacco Survey: Results of a split-ballot experiment
}

Neale Batra ${ }^{\star}$, Simone Gray ${ }^{\dagger}$ Nevin Krishna ${ }^{\ddagger}$, Nikhil Prachand ${ }^{\star \star}$, William Robinson ${ }^{\dagger \dagger}$, Cyprian Wejnert ${ }^{\ddagger \ddagger}$

Tags: survey practice

\section{Survey Practice}

Vol. 7, Issue 3, 2014

Response order effects in the Youth Tobacco Survey: Results of a split-ballot experiment

\section{INTRODUCTION}

Response order effects are changes in answers to close-ended questions that arise by varying the order of the response options (Krosnick and Alwin 1987). Two types of response order effects have been documented in previous studies: primacy effects and recency effects (Bishop and Smith 2001; Holbrook et al. 2007; Knauper 1999). Primacy effects, when response options presented earlier in a list of options are selected more often than ones at the end, have been typically observed in paper and pencil self-administered surveys (Krosnick and Alwin 1987). Recency effects, where response options presented later in a list are selected more often, primarily occur in surveys where questions are presented orally (Krosnick and Alwin 1987). Response order effects have been documented with adult respondents, but few studies have examined these effects with children or adolescents (Fuchs 2005). It is believed that no studies on response order effects have been conducted on a tobacco-specific survey.

Questionnaires often include non-substantive response options, such as "no opinion" or "don't know," to allow respondents who have no true opinions to select these options. People with lower education are more likely to be attracted to the "no opinion" response option (Krosnick et al. 2002). As a result, it is recommended that in children's surveys, researchers should minimize the use of non-substantive response categories (Bell 2007). However, in circumstances where it is not possible to avoid using non-substantive response options, no

\footnotetext{
Institution: Centers for Disease Control and Prevention, Division of HIV/AIDS Prevention; National Center for HIV/AIDS,

* Viral Hepatitis, STD and TB Prevention

Institution: Centers for Disease Control and Prevention, Division of HIV/AIDS Prevention; National Center for HIV/AIDS,

† Viral Hepatitis, STD and TB Prevention

Institution: Centers for Disease Control and Prevention, Division of HIV/AIDS Prevention; National Center for HIV/AIDS,

$\ddagger$ Viral Hepatitis, STD and TB Prevention

** Institution: Chicago Department of Public Health-STI/HIV Division

Institution: Louisiana Office of Public Health - STD/HIV Program; Louisiana State University Health Sciences Center in New

t† Orleans School of Public Health

Institution: Centers for Disease Control and Prevention, Division of HIV/AIDS Prevention; National Center for HIV/AIDS,

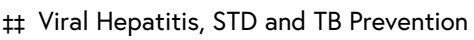


research has shown if the order of these response options has any effect on responses.

We assess whether primacy and/or recency effects occur in a self-administered tobacco-related questionnaire among youth and will determine the effects of reversing the order of response options and specifically changing the position of a non-applicable response category.

This study is one of very few studies to examine the effect of response order in children's responses, and it is the first to investigate the impact of moving a non-applicable response category from first to last position. To the best of our knowledge, it is also the first to document the presence of response order effects in a tobacco survey and differentiate the effects for tobacco users verses nonusers.

\section{METHODS}

\section{STUDY POPULATION AND SAMPLING METHODS}

The Youth Tobacco Survey (YTS) is conducted as a collaboration between the 50 states and the Centers for Disease Control and Prevention's Office on Smoking and Health. The YTS is administered to students in grades 6 through 121 and provides insight into the effectiveness of state tobacco control programs and measures the influence of pro-tobacco marketing and advertising on young people. The analysis combined YTS split-ballot surveys in Virginia and Mississippi. The Virginia survey was conducted between October 2007 and March 2008, and the Mississippi survey between January and August 2008. Regular public school students in 6th through 12th grade were eligible in Mississippi. Regular, alternative, or charter public school students were eligible in Virginia. ${ }^{1}$

Two-stage sample selection was used. In the first stage, schools were selected with probability proportional to enrollment. In Virginia, 50 high schools and 50 middle schools were sampled, and in Mississippi, 60 high schools and 60 middle schools were sampled. In the second stage, up to five classes in each school were selected, and the selected classes were randomly assigned to receive either the standard or test questionnaire.

All students in each selected class were eligible. From both states, 15,008 students were sampled, and 11,521 students participated. Forty-nine percent of the students were female, and 51 percent were male. The percentage of 11 year olds or younger, 12 year olds, 13 year olds, 14 year olds, 15 year olds, 16 year olds, 17 year olds, and 18 year olds or older were 7.4 percent, 13.6 percent,

\footnotetext{
1 The requirements for determining eligible schools, classes, and students are stated in The Youth Tobacco Survey Handbook. From the beginning, it has been standard practice to treat all partial interviews as completes. Denial of parental permission, absence from school, and refusals to participate constitute student nonresponse. Refusals are the only reason for school nonresponse. Schools that had closed since the beginning of the survey were considered ineligible.
} 
14.4 percent, 15.9 percent, 15.2 percent, 13.7 percent, 14.8 percent, and 4.8 percent, respectively. Forty-three percent of students were in middle school. Respectively, white and black students made up 55.4 percent and 33.3 percent of the population, and 5.7 percent were Hispanic and 5.6 percent were from other racial or ethnic groups.

School response rates were calculated by dividing the number of participating schools by the number of selected schools. Student response rates were calculated by dividing the number of participating students by the number of eligible students. The overall response rate is the product of these two rates. Overall response rates for the standard version were higher than the test version for high schools and middle schools in Virginia and Mississippi. The overall response rates for the standard vs. test versions in Virginia high schools, Virginia middle schools, Mississippi high schools, and Mississippi middle schools were 42.9 percent vs. 36.7 percent, 70.8 percent vs. 68.2 percent, 59.0 percent vs. 57.6 percent, and 65.3 percent vs. 61.8 percent, respectively.

\section{TYPES OF TESTS}

Within each state, middle and high school questionnaires were identical. The standard questionnaire contained 81 questions and the test questionnaire 82 test questions. The additional question in the test version was a result of splitting a question about race into two questions, one of which asked specifically about Hispanic ethnicity. Thirty-one questions involved primacy and recency tests (but not all 31 questions were asked in both states). ${ }^{2}$ We grouped these 31 questions into two categories based on the type of change to the response options:

1. Order-only tests:

In nine questions, we reversed response options in the test version. Two questions had three response options, six questions had four response options, and one question had five response options. Since we were testing for primacy or recency effects in these questions, we report changes in the distribution of responses from moving a response option from first to last position.

2. Non-applicable "NA" response option order tests:

The YTS questionnaire does not allow skip patterns. Non-tobacco users are required to answer questions about tobacco use, and a non-applicable (NA) response category was used to identify non-tobacco users. It also is used to identify other people for whom the question is non-applicable (such as young people who have not used the Internet and who must answer a question about Internet use). In 18 questions, we compared a standard version where the NA category was listed first with a test version where it was listed last. In four 
questions, the standard version NA category was listed last and the test version NA category first.

\section{STATISTICAL METHODS}

We calculated response estimates for each question adjusting for sample design effects. Raw percentage differences were calculated by subtracting standard-version percentages from test version percentages for each response category, and we used these percentage differences to determine primacy or recency effects. For example, in questions comparing a response option category that was first in the standard version and last in the test version, a negative percentage difference indicated a primacy effect while a positive difference indicated a recency effect. Rao-Scott and Wald Chi-Square tests were used to test statistical significance (_p_s $\leq 0.05)$. To allow for multiple comparisons, we adjusted alpha levels using Bonferroni criteria (Sedgwick 2012).

For each question, we used generalized multinomial or logistic regression models to determine if primacy or recency effects existed after controlling for covariates. The dependent variable in each model was the students' responses to the question. The exposure was the questionnaire version (standard or test). We controlled for age; school level (middle, high school); state (Mississippi, Virginia); and whether the student had been taught about tobacco in school, because some of the questions we analyzed asked about the students beliefs and attitudes towards tobacco. In four questions, changes were made between the standard and test version only in Mississippi, while in one other question, changes were only in Virginia's questionnaire. For these five questions, multinomial models only included data from the state where the changes were made. Predicted marginals were calculated for the questionnaire version variable (predicted marginals estimate the percentage of respondents for a selected group if everyone in the sample had been in that group). We calculated percentage differences of the predicted marginals, where the standard version predicted marginals were subtracted from the test-version predicted marginals.

Most of the questions that contained the NA tests had small sample sizes in many of the response categories. In those questions, the response options were collapsed, and the NA category was compared with all the other response options combined. In 12 NA questions about tobacco use, separate analyses were done for tobacco users and non-tobacco users. For the NA questions with a not applicable category of "I did not smoke cigarettes during the past 30 days," we analyzed those who had previously answered that they had smoked cigarettes during the past 30 days (tobacco users) separately from those who answered that they had not smoked cigarettes in the past 30 days, and we did the same for the questions on cigar and smokeless tobacco use. We did not perform adjusted tests in the analyses of tobacco and non-tobacco users responses because of the insufficient sample sizes that resulted from stratifying by tobacco use. 


\section{RESULTS}

In the order-only tests, all nine of the unadjusted tests exhibited primacy effects, meaning that in all nine questions respondents were more likely to choose a response option when it was first in a list. However, only three of the nine questions (33 percent) had statistically significant differences in the distribution of responses. The percentage differences for the first response category ranged from 0.8 to 6.9 percentage points, meaning that up to 6.9 percent more students chose a response category when it was first in the standard version than when it was last in the test version. From the adjusted multinomial models, eight of the nine questions exhibited primacy effects, but most questions did not have statistically significant differences between the standard and test versions (i.e., only two were statistically significantly different). Predicted marginal percentage differences ranged from 1.2 to 7.7 percentage points (Table 1). 
Table 1 Number and percentage of questions with primacy or recency effects by type of test.

\begin{tabular}{|c|c|c|c|c|c|}
\hline Types of test & Number of questions & Primacy effects $\mathrm{N}(\%)$ & $\begin{array}{l}\text { Recency } \\
\text { effects N (\%) }\end{array}$ & Significant ( $p \leq 0.05) \mathrm{N}(\%)$ & Range of percent differences \\
\hline \multicolumn{6}{|l|}{ Order tests } \\
\hline Unadjusted & 9 & $9(100.0)$ & $0(0.0)$ & $3(33.3)$ & $(0.8-6.9)^{*}$ \\
\hline Adjusted ${ }^{\dagger}$ & 9 & $8(88.9)$ & $1(11.0)$ & $2(22.2)$ & $(1.2-7.7)^{*}$ \\
\hline \multicolumn{6}{|l|}{ NA tests } \\
\hline Unadjusted & 22 & $21(95.5)$ & $1(4.5)$ & $3(13.6)$ & $(0.4-6.3)$ \\
\hline Adjusted ${ }^{\dagger}$ & 22 & $21(95.5)$ & $1(4.5)$ & $3(13.6)$ & $(0.9-6.2)$ \\
\hline \multicolumn{6}{|l|}{ NA tests } \\
\hline Tobacco users (unadjusted) & 12 & $3(25.0)$ & $9(75.0)$ & $6(50.0)$ & $(0.3-12.0)$ \\
\hline Non-tobacco users (unadjusted) & 12 & $11(91.7)$ & $1(8.3)$ & $10(83.3)$ & $(0.9-4.2)$ \\
\hline
\end{tabular}

${ }^{*}$ Range for the first response option.

$\lceil$ Adjusted for age, school level, state, and whether the student had been taught about tobacco in school. 
In both the unadjusted and adjusted non-applicable response option order tests, 96 percent of the questions (21 out of 22) showed primacy effects and one question had a recency effect. Three had statistically significant primacy effects in both the unadjusted tests and the adjusted models. In the unadjusted tests, shifts in the distribution of responses ranged from 0.4 to 6.3 percentage points, and in the adjusted tests, from 0.9 to 6.2 percentage points (Table 1 ).

When we analyzed tobacco users' responses to 12 "NA" response option order tests among tobacco users, recency effects were present in nine of the questions and primacy effects occurred in three questions. Six of the 12 questions had significant differences; three of those with recency effects and all three questions with primacy effects had significant differences. Shifts in the distribution of responses ranged from 0.3 to 12.0 percentage points. When we analyzed non-tobacco users responses to these same questions, primacy effects were present in all but one question that had a recency effect, and 10 (83 percent) of questions had significant differences (nine primacy effects and one recency effect).

\section{DISCUSSION}

Our results support the hypothesis that children are prone to primacy effects in self-administered questionnaires. Questions with a non-applicable response option that also is the first option are vulnerable to primacy effects. This finding is analogous to findings among adult respondents who tend to favor non-substantive options over substantive ones (Krosnick et al. 2002). In our study, students were more likely to select the non-applicable response option when it was first in a list of options as opposed to last. Also, although we did not measure the effect of each question's complexity, the complexity may be important; for example, children may be more prone to primacy effects in questions with complex response options. Many of the questions with significant effects had several (up to 7 ) response options (Table 2), and previous studies have demonstrated that 7 or more response options decreases scale reliability, with 4 response options being the "optimal" number for children and adolescents (Borgers et al. 2004). Therefore, it is possible that there are significant differences in these questions because of the number of response options. 
Table 2 Questions with significant* primacy or recency effects.

\begin{tabular}{|c|c|c|}
\hline Question order & Question & Type of effect \\
\hline 12 & During the past 30 days, on the days you smoked, how many cigarettes did you smoke per day? & Both $^{\dagger}$ \\
\hline 13 & During the past 30 days, what brands of cigarettes did you smoke? (You can CHOOSE ONE ANSWER or MORE THAN ONE ANSWER) & Both \\
\hline $14^{\S}$ & During the past 30 days, what brand of cigarettes did you usually smoke? (CHOOSE ONLY ONE ANSWER & Both \\
\hline $14 \pi$ & During the past 30 days, what brand of cigarettes did you usually smoke? (CHOOSE ONLY ONE ANSWER) & Primacy \\
\hline 16 & During the past 30 days, how did you get cigarettes? (You can CHOOSE ONE ANSWER or MORE THAN ONE ANSWER) & Primacy \\
\hline 17 & During the past 30 days, how did you usually get cigarettes? (CHOOSE ONLY ONE ANSWER) & Both \\
\hline 30 & During the past 12 months, did you ever try to quit smoking cigarettes? & Primacy \\
\hline 34 & How old were you when you used chewing tobacco, snuff, or dip for the first time? & Primacy \\
\hline 37 & During the past 30 days, how did you get chewing tobacco, snuff, or dip? (You can CHOOSE ONE ANSWER or MORE THAN ONE ANSWER) & Primacy \\
\hline 38 & During the past 30 days, how did you usually get chewing tobacco, snuff, or dip? (CHOOSE ONLY ONE ANSWER) & Primacy \\
\hline 40 & How old were you when you smoked a cigar, cigarillo, or little cigar for the first time? & Primacy \\
\hline 62 & When you watch TV or go to movies, how often do you see actors using tobacco? & Primacy \\
\hline 71 & What do you think people should do about smoking inside their home? People should ... & Primacy \\
\hline 73 & What do you think people should do about smoking in their vehicles? People should ... & Primacy \\
\hline
\end{tabular}

*Either significant in the adjusted overall models or significant among tobacco or non-tobacco users

† "Both" indicates a primacy effect and recency effect (a recency effect among tobacco users and a primacy effect among nontobacco users or vice versa)

§Mississippi only

gVirginia only 
When we analyzed tobacco users' responses to NA questions about tobacco use, primacy effects were observed for three questions, and recency effects were observed for three questions. We were interested in how tobacco users answered these particular questions, since tobacco users choosing the inappropriate category is an indication of inconsistency in their responses throughout the survey. We also analyzed non-tobacco users' responses in the same tobacco use questions and found that non-tobacco users were more prone to primacy effects. This means that the findings for non-tobacco users did not differ much from the findings overall, which we would have expected since non-tobacco users make up the majority of the respondents for the NA category. Also, non-tobacco users need to select the NA category for many questions, and after doing so for several questions, perhaps some would assume that the survey does not apply to them, making them more likely to satisfice and select the NA option every time, especially when it is conveniently listed first.

Our study had several limitations. Our results were limited to students in just two states, and overall high school response rates in Virginia were very low (36.7 percent). Another limitation is that we did not use the same students as the comparison group by conducting both sets of surveys on the same students. Also, we made multiple comparisons, and even though we used Bonferroni corrections, it is possible that significant differences were due to chance.

On the basis of the findings, we believe that more research is needed to duplicate our results, especially in the area of the NA category where we found a discrepancy between tobacco and non-tobacco users.

\section{CONCLUSION}

In our YTS split-ballot experiment, we observed primarily primacy effects and a few recency effects (primarily among tobacco users) even after controlling for age, school level, state, and whether the student had been taught about tobacco in school. We also concluded that the position of a non-applicable response option does have an effect on responses, especially among non-tobacco users, and respondents will be prone to select this option more often when it is first as opposed to last in a list of options.

Consistent with the previous research, the findings from our study suggest that rotating the order of response options, so that different respondents receive different orders, may be beneficial for tobacco-related youth surveys. This approach could be viable for electronic data collection, as well as for a paper-and-pencil questionnaire such as the YTS, where researchers could provide two versions of the questionnaire in each school. To date, the majority of survey organizations do not rotate the order of response options (Holbrook et al. 2007).

Also consistent with general survey design principals, it is important to consider using skip patterns instead of a "not applicable" response option 
(Fisher 2000). In situations where skip patterns cannot be used, "not applicable" is best positioned as the last response option, rather than the first, so as to ensure that a greater percentage of respondents will select substantive options.

\section{AUTHOR NOTE}

The findings and conclusions in this report are those of the authors and do not necessarily represent the official position of the Centers for Disease Control and Prevention.

\section{ACKNOWLEDGEMENTS}

Jennifer Bombard, Peter Mariolis, Valerie J. Rock, Heather Ryan, Stacy Thorne

\section{REFERENCES}

Bell, A. 2007. Designing and testing questionnaires for children. Journal of Research in Nursing 12(5): 461-469.

Bishop, G. and A. Smith. 2001. Response-order effects and the early Gallup split-ballots. Public Opinion Quarterly 65(4): 479-505.

Borgers, N., J. Hox and D. Sikkel. 2004. Response effects in surveys on children and adolescents: The effect of number of response options, negative wording, and neutral mid-point. Quality E'Quantity 38(1): 17-33.

Fisher, W.P. 2000. Survey design recommendations. Popular Measurement 3(1): 58-59.

Fuchs, M. 2005. Children and adolescents as respondents. Experiments in question order, response order, scale effects and the effect of numeric values associated with response options. Journal of Official Statistics 21(4): 701-725.

Holbrook, A.L., J.A. Krosnick, D. Moore and R. Tourangeau. 2007. Response order effects in dichotomous categorical questions presented orally - The impact of question and respondent attributes. Public Opinion Quarterly 71(3): 325-348.

Knauper, B. 1999. The impact of age and education on response order effects in attitude measurement. Public Opinion Quarterly 63(3): 347-370.

Krosnick, J.A. and D.F. Alwin. 1987. An evaluation of a cognitive theory of response-order effects in survey measurement. Public Opinion Quarterly 51(2): 201-219.

Krosnick, J.A., A.L. Holbrook, M.K. Berent, R.T. Carson, W.M. Hanemann, R.J. Kopp, R.C. Mitchell, S. Presser, P.A. Ruud, V.K. Smith, W.R. Moody, M.C. Green and M. Conaway. 2002. The impact of "no opinion" response options on data quality - Non-attitude reduction or an invitation to satisfice? 
Public Opinion Quarterly 66(3): 371-403.

Sedgwick, P. 2012. Statistical Question Multiple significance tests: the Bonferroni correction. British Medical Journal 344: e509.

\section{SUPPLEMENTAL MATERIAL}

\section{YTS Questions, Standard and Test Versions for Order-only Response Option Tests.}

\section{Standard Version Question}

1. Do you think you will smoke a cigarette at any time during the next year?

a. Definitely yes

b. Probably yes

c. Probably not

d. Definitely not

2. If one of your best friends offered you a cigarette, would you smoke it?

a. Definitely yes

b. Probably yes

c. Probably not

d. Definitely not

3. In the past 12 months, how often have your parents or guardians discussed the dangers of tobacco use with you? a. Never

b. Rarely

c. Sometimes

d. Often

e. Very often

4. Do you think young people who smoke cigarettes have more friends?

a. Definitely yes

b. Probably yes

c. Probably not

d. Definitely not

5. Do you think smoking cigarettes makes young people look cool or fit in?

a. Definitely yes

b. Probably yes

c. Probably not

d. Definitely not

6. Do you think it is safe for young people to smoke cigarettes as long as they don't smoke every day?*

a. Definitely yes

b. Probably yes

c. Probably not

d. Definitely not

7. Do you think that tobacco companies try to get young people to use tobacco products?

a. Definitely yes

b. Probably yes

c. Probably not

d. Definitely not

8. What do you think people should do about smoking inside their home? People should ...*

a. Never allow smoking inside their home

b. Allow smoking at some times or in some places

c. Always allow smoking inside their home
Test Version Question

1. Do you think you will smoke a cigarette at any time during the next year?

a. Definitely not

b. Probably not

c. Probably yes

d. Definitely yes

2. If one of your best friends offered you a cigarette, would you smoke it?

a. Definitely not

b. Probably not

c. Probably yes

d. Definitely yes

3. In the past 12 months, how often have your parents or guardians discussed the dangers of tobacco use with you? a. Very often

b. Often

c. Sometimes

d. Rarely

e. Never

4. Do you think young people who smoke cigarettes have more friends?

a. Definitely not

b. Probably not

c. Probably yes

d. Definitely yes

5. Do you think smoking cigarettes makes young people look cool or fit in?

a. Definitely not

b. Probably not

c. Probably yes

d. Definitely yes

6. Do you think it is safe for young people to smoke cigarettes as long as they don't smoke every day?*

a. Definitely not

b. Probably not

c. Probably yes

d. Definitely yes

7. Do you think that tobacco companies try to get young people to use tobacco products?

a. Definitely not

b. Probably not

c. Probably yes

d. Definitely yes

8. What do you think people should do about smoking inside their home? People should ...*

a. Always allow smoking inside their homes

b. Allow smoking at some times or in some places

c. Never allow smoking inside their home

* Only asked in Mississippi 
YTS Questions, Standard and Test Versions for Not Applicable Response Option Tests.

1. How old were you when you smoked a whole cigarette for the first time?

a. I have never smoked a whole cigarette

b. 8 years old or younger

c. 9 or 10 years old

d. 11 or 12 years old

e. 13 or 14 years old

f. 15 or 16 years old

g. 17 years old or older

2. During the past 30 days, on the days you smoked, how many cigarettes did you smoke per day?

a. I did not smoke cigarettes during the past 30 days

b. Less than 1 cigarette per day

c. 1 cigarette per day

d. 2-5 cigarettes per day

e. 6-10 cigarettes per day

f. 11-20 cigarettes per day

g. More than 20 cigarettes per day

3. During the past 30 days, what brands of cigarettes did you smoke? (You can CHOOSE ONE ANSWER or MORE THAN

ONE ANSWER)

a. I did not smoke cigarettes during the past 30 days

b. American Spirit

c. Camel

d. GPC, Basic or Doral

e. Kool

f. Marlboro

g. Newport

h. Some other brand not listed here

4. During the past 30 days, what brand of cigarettes did you usually smoke? (CHOOSE ONLY ONE ANSWER)*

a. I did not smoke cigarettes during the past 30 days

b. American Spirit

c. Camel

d. GPC, Basic or Doral

e. Kool

f. Marlboro

g. Newport

h. Some other brand not listed here

5. During the past 30 days, what brand of cigarettes did you usually smoke? (CHOOSE ONLY ONE ANSWER) ${ }^{\dagger}$

a. American Spirit

b. Camel

c. GPC, Basic or Doral

d. Kool

e. Marlboro

f. Newport

g. Some other brand not listed here

h. I did not smoke cigarettes during the past 30 days

6. During the past 30 days, how did you get cigarettes? (You can CHOOSE ONE ANSWER or MORE THAN ONE ANSWER)

a. I did not smoke cigarettes during the past 30 days

b. I bought them myself

c. I gave someone else money to buy them for me

d. I borrowed (or bummed) them

e. Someone gave them to me

f. I took them from a store or another person

g. I got them some other way

7. During the past 30 days, how did you usually get cigarettes? (CHOOSE ONLY ONE ANSWER)

a. I did not smoke cigarettes during the past 30 days

b. I bought them myself

c. I gave someone else money to buy them for me

d. I borrowed (or bummed) them

e. Someone gave them to me

f. I took them from a store or another person

g. I got them some other way
1. How old were you when you smoked a whole cigarette for the first time?

a. 8 years old or younger

b. 9 or 10 years old

c. 11 or 12 years old

d. 13 or 14 years old

e. 15 or 16 years old

f. 17 years old or older

g. I have never smoked a whole cigarette

2. During the past 30 days, on the days you smoked, how many cigarettes did you smoke per day?

a. Less than 1 cigarette per day

b. 1 cigarette per day

c. $2-5$ cigarettes per day

d. $6-10$ cigarettes per day

e. 11-20 cigarettes per day

f. More than 20 cigarettes per day

g. I did not smoke cigarettes during the past 30 days

3. During the past 30 days, what brands of cigarettes did you smoke? (You can CHOOSE ONE ANSWER or MORE THAN

ONE ANSWER)

a. American Spirit

b. Camel

c. GPC, Basic or Doral

d. Kool

e. Marlboro

f. Newport

g. Some other brand not listed here

h. I did not smoke cigarettes during the past 30 days

4. During the past 30 days, what brands of cigarettes did you usually smoke? (CHOOSE ONLY ONE ANSWER)*

a. American Spirit

b. Camel

c. GPC, Basic or Doral

d. Kool

e. Marlboro

f. Newport

g. Some other brand not listed here

h. I did not smoke cigarettes during the past 30 days

5. During the past 30 days, what brand of cigarettes did you usually smoke? (CHOOSE ONLY ONE ANSWER) ${ }^{\dagger}$

a. I did not smoke cigarettes during the past 30 days

b. American Spirit

c. Camel

d. GPC, Basic or Doral

e. Kool

f. Marlboro

g. Newport

h. Some other brand not listed here

6. During the past 30 days, how did you get cigarettes? (You can CHOOSE ONE ANSWER or MORE THAN ONE ANSWER)

a. I bought them myself

b. I gave someone else money to buy them for me

c. I borrowed (or bummed) them

d. Someone gave them to me

e. I took them from a store or another person

f. I got them some other way

g. I have not smoked cigarettes during the past 30 days

7. During the past 30 days, how did you usually get cigarettes? (CHOOSE ONLY ONE ANSWER)

a. I bought them myself

b. I gave someone else money to buy them for me

c. I borrowed (or bummed) them

d. Someone gave them to me

e. I took them from a store or another person

f. I got them some other way

g. I did not smoke cigarettes during the past 30 days 
8. How long can you go without smoking before you feel like you need a cigarette?

a. I have never smoked cigarettes

b. I do not smoke now

c. Less than an hour

d. 1-3 hours

e. More than 3 hours but less than a day

f. A whole day

g. Several days

h. A week or more

9. During the past 12 months, did you ever try to quit smoking cigarettes?

a. I did not smoke during the past 12 months

b. Yes

c. No

10. How old were you when you used chewing tobacco, snuff, or dip for the first time?

a. I have never used chewing tobacco, snuff, or dip

b. 8 years old or younger

c. 9 or 10 years old

d. 11 or 12 years old

e. 13 or 14 years old

f. 15 or 16 years old

g. 17 years old or older

11. During the past 30 days, how did you get chewing tobacco, snuff, or dip? (You can CHOOSE ONE ANSWER or MORE THAN ONE ANSWER)

a. I did not use chewing tobacco, snuff, or dip during the past 30 days

b. I bought it myself

c. I gave someone else money to buy it for me

d. I borrowed (or bummed) it

e. Someone gave it to me

f. I took it from a store or another person

g. I got it another way

12. During the past 30 days, how did you usually get chewing tobacco, snuff, or dip? (CHOOSE ONLY ONE ANSWER)

a. I did not use chewing tobacco, snuff, or dip during the past 30 days

b. I bought it myself

c. I gave someone else money to buy it for me

d. I borrowed (or bummed) it

e. Someone gave it to me

f. I took it from a store or another person

g. I got it some other way

13. How old were you when you smoked a cigar, cigarillo, or little cigar for the first time?

a. I have never smoked a cigar, cigarillo, or little cigar

b. 8 years old or younger

c. 9 or 10 years old

d. 11 or 12 years old

e. 13 or 14 years old

f. 15 or 16 years old

g. 17 years old or older

14. During the past 30 days, how did you get cigars, cigarillos, or little cigars? (You can CHOOSE ONE ANSWER or MORE THAN ONE ANSWER)

a. I did not smoke cigars, cigarillos, or little cigars during the past 30 days

b. I bought them myself

c. I gave someone else money to buy them for me

d. I borrowed (or bummed) them

e. Someone gave them to me

f. I took them from a store or another person

g. I got them some other way

15. During the past 30 days, how did you usually get cigars, cigarillos, or little cigars? (CHOOSE ONLY ONE ANSWER)

a. I did not smoke cigars, cigarillos, or little cigars during the
8. How long can you go without smoking before you feel like you need a cigarette?

a. Less than an hour

b. 1-3 hours

c. More than 3 hours but less than a day

d. A whole day

e. Several days

f. A week or more

g. I have never smoked cigarettes

h. I do not smoke now

9. During the past 12 months, did you ever try to quit smoking cigarettes?

a. Yes

b. No

c. I did not smoke during the past 12 months

10. How old were you when you used chewing tobacco, snuff, or dip for the first time?

a. 8 years old or younger

b. 9 or 10 years old

c. 11 or 12 years old

d. 13 or 14 years old

e. 15 or 16 years old

f. 17 years old or older

g. I have never used chewing tobacco, snuff, or dip

11. During the past 30 days, how did you get chewing tobacco, snuff, or dip? (You can CHOOSE ONE ANSWER or MORE

THAN ONE ANSWER)

a. I bought it myself

b. I gave someone else money to buy it for me

c. I borrowed (or bummed) it

d. Someone gave it to me

e. I took it from a store or another person

f. I got it some other way

g. I did not use chewing tobacco, snuff, or dip during the past 30 days

12. During the past 30 days, how did you usually get chewing tobacco, snuff, or dip? (CHOOSE ONLY ONE ANSWER)

a. I bought it myself

b. I gave someone else money to buy it for me

c. I borrowed (or bummed) it

d. Someone gave it to me

e. I took it from a store or another person

f. I got it some other way

g. I did not use chewing tobacco, snuff, or dip during the past 30 days

13. How old were you when you smoked a cigar, cigarillo, or little cigar for the first time?

a. 8 years old or younger

b. 9 or 10 years old

c. 11 or 12 years old

d. 13 or 14 years old

e. 15 or 16 years old

f. 17 years old or older

g. I have never smoked a cigar, cigarillo or little cigar

14. During the past 30 days, how did you get cigars, cigarillos, or little cigars? (You can CHOOSE ONE ANSWER or MORE THAN ONE ANSWER)

a. I bought them myself

b. I gave someone else money to buy them for me

c. I borrowed (or bummed) them

d. Someone gave them to me

e. I took them from a store or another person

f. I got them some other way

g. I did not smoke cigars, cigarillos, or little cigars during the past 30 days

15. During the past 30 days, how did you usually get cigars, cigarillos, or little cigars? (CHOOSE ONLY ONE ANSWER) a. I bought them myself 
past 30 days

b. I bought them myself

c. I gave someone else money to buy them for me

d. I borrowed (or bummed) them

e. Someone gave them to me

f. I took them from a store or another person

g. I got them some other way

16. Have you ever tried smoking tobacco in a pipe or water pipe?

a. Pipe

b. Water pipe

c. Both

d. I have never smoked tobacco in a pipe or water pipe

17. Have you ever tried smoking any of the following:

a. Bidis

b. Kreteks

c. I have tried both bidis and kreteks

d. I have never smoked bidis or kreteks

18. Do you think that you will try a cigarette soon? ${ }^{\dagger}$

a. I have already tried smoking cigarettes

b. Yes

c. No

19. Have you ever participated in a program to help you quit using tobacco?

a. I have never used tobacco

b. Yes

c. No

20. During the past 12 months, have you participated in any community activities to discourage people your age from using cigarettes, chewing tobacco, snuff, dip, or cigars?

a. Yes

b. No

c. I did not know about any activities

21. When you watch TV or go to movies, how often do you see actors using tobacco?

a. I don't watch TV or go to movies

b. Most of the time

c. Some of the time

d. Hardly ever

e. Never

22. When you are using the Internet, how often do you see ads for tobacco products?

a. I don't use the Internet

b. Most of the time

c. Some of the time

d. Hardly ever

e. Never

*Only asked in Virginia

†Only asked in Mississippi b. I gave someone else money to buy them for me

c. I borrowed (or bummed) them

d. Someone gave them to me

e. I took them from a store or another person

f. I got them some other way

g. I did not smoke cigars, cigarillos, or little cigars during the past 30 days

16. Have you ever tried smoking tobacco in a pipe or water pipe?

a. I have never smoked tobacco in a pipe or water pipe

b. Pipe

c. Water pipe

d. Both

17. Have you ever tried smoking any of the following:

a. I have never smoked bidis or kreteks

b. Bidis

c. Kreteks

d. I have tried both bidis and kreteks

18. Do you think that you will try a cigarette soon? ${ }^{\dagger}$

a. Yes

b. No

c. I have already tried smoking cigarettes

19. Have you ever participated in a program to help you quit using tobacco?

a. Yes

b. No

c. I have never used tobacco

20. During the past 12 months, have you participated in any community activities to discourage people your age from using cigarettes, chewing tobacco, snuff, dip, or cigars?

a. I did not know about any activities

b. Yes

c. No

21. When you watch TV or go to movies, how often do you see actors using tobacco?

a. Most of the time

b. Some of the time

c. Hardly ever

d. Never

e. I don't watch TV or go to movies

22. When you are using the Internet, how often do you see ads for tobacco products?

a. Most of the time

b. Some of the time

c. Hardly ever

d. Never

e. I don't use the Internet 